gave it to a nurse or doctor on the ward. The nurse and doctor would consider the suggested solution and tick off either 'Yes I agree and have prescribed a laxative' or 'No I don't agree' and return the handout to the pharmaconomist.

Results A total of 2282 patient medicines were screened and 681 patients had been prescribed opioids. 236 of the patients receiving opioids did not have a prescription for a laxative and the pharmaconomist filled in an intervention handout for these patients. 25 interventions were accepted by the doctors on the wards and laxatives were prescribed. Unfortunately about $50 \%$ of the handouts were never returned to the pharmacy, making it difficult to determine the exact number of interventions accepted. Also a number of patients were discharged before action could be taken. If the intervention were to be repeated the following would be relevant to improve the outcome: better communication with the doctors, ensure that the patients on selected wards are hospitalised for a few days (to make time for intervention) and more time to prepare the ward personnel. Conclusions The campaign was a success, but more could be done to improve the outcomes of such a campaign. It is important to consider the selection of wards to include in the campaign. On wards where the patients are discharged after a few days it can be difficult to implement the interventions under time pressure.

No conflict of interest.

\section{CPC-117 PROSPECTIVE REGISTRY FOR EVALUATING THE EFFECTIVENESS OF BEVACIZUMAB ALONE OR WITH IRINOTECAN IN RECURRENT GLIOBLASTOMA}

doi:10.1136/ejhpharm-2013-000276.574

${ }^{1} \mathrm{M}$ Vaiani, ${ }^{1} \mathrm{M}$ Cecchi, 'S Colombini, 'E Agostino, 'P Attanasio, ${ }^{2} \mathrm{M}$ Ceroti, 'R Banfi. ${ }^{1}$ Careggi Hospital, Pharmacy Department, Florence, Italy; ${ }^{2}$ ISPO, Molecular and Nutritional Epidemiology Unit, Florence, Italy

Background Recurrent glioblastoma is nearly always fatal, with median survival rates of approximately 12-14 months. Previous phase II clinical trials showed promising results with bevacizumab, alone or in combination with irinotecan, in patients with recurrent glioblastoma.

Purpose To assess whether the survival of patients with recurrent glioblastoma receiving bevacizumab alone or with irinotecan in everyday practise is comparable to that reported in clinical trials.

Materials and Methods This was a retrospective observational study conducted at a single hospital in Italy. Patients with recurrent glioblastoma who had received bevacizumab alone or with irinotecan from January 2009 to September 2011 were included in our study.

The main outcome measures were progression-free survival (PFS), overall survival (OS), and rates of PFS and OS at 6 months. Results Median PFS was 5.1 months in the bevacizumab group $(\mathrm{n}=9)$ and 15.4 months in the bevacizumab + irinotecan group $(n=10)$, with 6 -month PFS rates of $45 \%$ and $69 \%$, respectively. Median OS was 6.8 months for bevacizumab alone and 11.1 months for bevacizumab + irinotecan, with 6-month OS rates of $100 \%$ and $90 \%$, respectively.

Conclusions Although the number of patients included is not sufficient to allow a conclusive statement about the place of bevacizumab in the treatment of recurrent glioblastoma, the data appear promising, and are consistent with the results of clinical trials.

No conflict of interest.

\section{CPC-118 QUICK WINS - INNOVATIVE AND ECONOMIC FOCUS ON USE OF MEDICINES}

\section{doi:10.1136/ejhpharm-2013-000276.575}

${ }^{1}$ A Kobberø Jensen, 'D Vilstrup Tomsen, ${ }^{2 B}$ Ørskov Lindhardt. 'Region Hovedstadens Apotek, Clinical Pharmacy Services, Hillerød, Denmark; ${ }^{2}$ Hillerød Hospital, Lung- and Infection, Hillerød, Denmark
Background Medicines account for a large part of the budget in Danish hospitals. National and regional actions are initiated to control drug expenses. Local initiatives aim at involving doctors, nurses and pharmacy staff in taking everyday responsibility for the rational use of drugs.

Purpose To establish systematic and documented cooperation between the Local Drug and Therapeutics Committee, the clinical staff and the pharmacy staff to systematically identify and intervene on avoidable medicines expenses.

Materials and Methods 10 focus areas were identified: Handling free-of-charge drugs, systematic feedback from top-up-service, the staff's (unofficial) use of medicines, reanalysis of statistical material on drug use, input from other pharmacy departments, analysing the use of the 120 most expensive drugs, analysis of disposed medicines, emptying vials (expensive drugs), shift from IV to oral antibiotics, and use of the patients' own medicine, when possible. Through a systematic approach and co-operation on all levels of the organisation, the 10 focus areas were implemented in everyday practise at the hospital.

Results The results were recorded in a report to the Local Drug and Therapeutics Committee in January 2012. A financial estimate was made for 4 out of 10 focus areas. The total result for the 4 intervention areas amounts to a saving of DKK 1,154,500 $(€ 155,00) /$ year. The saving is based on a conservative estimate. For the remaining six focus areas interventions are still taking place. In 2012 the initiative is spreading to other hospitals in the Capital Region.

Conclusions Through systematic and well-documented cooperation between the Local Drug and Therapeutics Committee, the clinical staff and the pharmacy, it has proved possible to save a considerable amount on the total hospital budget.

No conflict of interest.

\section{CPC-119 RANDOMIZED CONTROLLED TRIAL OF CLINICAL PHARMACY MANAGEMENT OF PATIENTS WITH TYPE 2 DIABETES IN AN OUTPATIENT DIABETES CLINIC IN JORDAN}

doi:10.1136/ejhpharm-2013-000276.576

${ }^{1}$ A Jarab, ${ }^{2} \mathrm{~S}$ Alqudah, ${ }^{3} \mathrm{~T}$ Mukattash, ${ }^{1} \mathrm{G}$ Shattat, ${ }^{1} \mathrm{~T}$ Al-Qirim. ${ }^{1}$ Alzaytoonah University of Jordan, Pharmacy, Amman, Jordan; 'Jordanian Royal Medical Services, Pharmacy, Amman, Jordan; ${ }^{3}$ Jordan University of Science and Technology, Pharmacy, Irbid, Jordan

Background Glycaemic goals are often not achieved in patients with type 2 diabetes despite the availability of many effective treatments and the documented benefits of glycaemic control in the reduction of long-term microvascular and macrovascular complications.

Purpose To evaluate, in a randomised, controlled trial, the impact of a clinical pharmacy service on clinical outcomes in patients with type 2 diabetes.

Materials and Methods A total of 171 patients (85 interventions vs. 86 usual care) participated in the study. Intervention patients had individualised education and treatment recommendations from a clinical pharmacist while control patients received usual care provided by the clinic. The primary outcome measure was glycaemic control manifested by HbA1c reductions. All other data collected including systolic and diastolic blood pressure, total cholesterol, low density lipoprotein (LDL), high density lipoprotein (HDL), serum triglycerides, medication adherence, and necessary self-care activities formed secondary outcome measures. Between-group differences in the amounts of change from baseline to 6-month follow-up were tested and a $p$ value of $<0.05$ was considered statistically significant.

Results Compared with baseline values, patients in the intervention group had a mean reduction of $0.8 \%$ in $\mathrm{HbA1c}$ versus a mean 